\title{
Classical and quantum entropy of parton distributions
}

\author{
Yoshikazu Hagiwara, ${ }^{1}$ Yoshitaka Hatta, ${ }^{2}$ Bo-Wen Xiao, ${ }^{3}$ and Feng Yuan ${ }^{4}$ \\ ${ }^{1}$ Department of Physics, Kyoto University, Kyoto 606-8502, Japan \\ ${ }^{2}$ Yukawa Institute for Theoretical Physics, Kyoto University, Kyoto 606-8502, Japan \\ ${ }^{3}$ Key Laboratory of Quark and Lepton Physics (MOE) and Institute of Particle Physics, Central China \\ Normal University, Wuhan 430079, China \\ ${ }^{4}$ Nuclear Science Division, Lawrence Berkeley National Laboratory, Berkeley, California 94720, USA
}

(Received 28 March 2018; published 29 May 2018)

\begin{abstract}
We introduce the semiclassical Wehrl entropy for the nucleon as a measure of complexity of the multiparton configuration in phase space. This gives a new perspective on the nucleon tomography. We evaluate the entropy in the small- $x$ region and compare with the quantum von Neumann entropy. We also argue that the growth of entropy at small $x$ is eventually slowed down due to the Pomeron loop effect.
\end{abstract}

DOI: 10.1103/PhysRevD.97.094029

\section{INTRODUCTION}

In the study of the partonic structure of hadrons, the methods of thermodynamics and statistical physics have often turned out to be useful. For example, there exist parametrizations of the parton distribution functions (PDFs) inspired by the Bose-Einstein and Fermi-Dirac distributions [1-3]. Another example is that the energy evolution of the density of soft gluons has been treated as a reaction-diffusion process in statistical physics [4,5]. Intuitively, if there is a multitude of partons involved in a given process, it is reasonable to expect that certain features of observables admit a simple statistical description. The picture becomes increasingly more attractive at very high energy or in the small- $x$ region where the number of gluons grows rapidly.

In recent years, several authors have introduced the notion of entropy of small- $x$ gluons in the hadron wave function and discussed its connection to the multiplicity in the final state [6-11] (see also [12-14]). A hadron in its ground state is a pure quantum state for which the standard quantum (von Neumann) entropy vanishes. Yet, one can think of various types of partons with different values of $x$ as different subsystems which are entangled to each other. As experiment can only probe a small part of the hadron wave function above a certain value of $x$ while the rest is being integrated, an entanglement entropy may be defined and calculated. However, previous discussions along this line relied on particular formalisms at small $x$ (the color

Published by the American Physical Society under the terms of the Creative Commons Attribution 4.0 International license. Further distribution of this work must maintain attribution to the author(s) and the published article's title, journal citation, and DOI. Funded by SCOAP. glass condensate formalism [9] and the dipole formalism [10]) which cannot be straightforwardly generalized to the quark sector or to the large- $x$ region. It would be interesting to have a more accessible, model-independent definition of entropy in terms of the quark and gluon field operators so that it can be analyzed by various perturbative and nonperturbative means.

In this paper, we study the entropy of quarks and gluons defined through the QCD Wigner [15] and Husimi [16] distributions. These are multidimensional phase space distributions of quarks and gluons inside a hadron, and have been actively pursued in the context of nucleon tomography [17-31]. In fact, it is quite natural to define an entropy via phase space distributions. In quantum mechanics, the corresponding construction is known as the Wehrl entropy [32] which is a semiclassical counterpart of the fully quantum von Neumann entropy. The Wehrl entropy has been previously discussed in QCD in [33-35] for a different purpose (the problem of thermalization in heavy-ion collisions) with a totally different definition. The present definition, appropriate for the study of the nucleon structure, was briefly given in [36], but was not explored. Here, we give a general discussion of the Wehrl entropy associated with the QCD Husimi distribution [23] and evaluate it in the small- $x$ region in a model that features the gluon saturation effects. We also extend the result of [10] by including the so-called Pomeron loop effect and demonstrate that this leads to the saturation of entropy at small $x$.

\section{ENTROPY IN CLASSICAL AND QUANTUM SYSTEMS}

In this section, we briefly review the definitions of entropy in classical and quantum mechanics. For simplicity, we consider a one-dimensional system, but generalization 
to arbitrary dimensions is straightforward. In statistical physics and kinetic theory, entropy is defined via the phase space distribution function $f(q, p)(q, p$ are the coordinate and momentum of particles) as

$$
S_{c l}=-\int \frac{d q d p}{2 \pi \hbar} f(q, p) \ln f(q, p) .
$$

If the system is out of equilibrium, $f$ depends on time $t$ according to the Boltzmann equation $\frac{d}{d t} f=C[f]$. As is well known, $S_{c l}(t)$ increases monotonically and eventually saturates as the system reaches equilibrium.

For a quantum system, the usual definition of entropy is the von Neumann entropy,

$$
S_{v N}=-\operatorname{Tr} \rho \ln \rho,
$$

where $\rho$ is the density matrix. For a pure state $\rho=|\psi\rangle\langle\psi|$, $S_{v N}$ vanishes. A nonzero $S_{v N}$ then measures the degree of deviation from a pure state. For a density matrix of the form $\rho=\sum_{n} p_{n}\left|\psi_{n}\right\rangle\left\langle\psi_{n}\right|$, it is given by

$$
S_{v N}=-\sum_{n} p_{n} \ln p_{n} .
$$

The two entropies $S_{c l}$ and $S_{v N}$ are not simply related to each other. In particular, the latter does not reduce to the former in the limit $\hbar \rightarrow 0$. To make a connection between the two, Wehrl introduced an intermediate definition of entropy [32]. Let $|\lambda\rangle$ with $\lambda=\frac{1}{\sqrt{2 \hbar}}(q+i p)$ be the coherent state which is the eigenstate of the annihilation operator $a|\lambda\rangle=\lambda|\lambda\rangle$. Taking the trace in (2) in the coherent state basis, one gets

$$
S_{v N}=-\int \frac{d q d p}{2 \pi \hbar}\langle\lambda|\rho \ln \rho| \lambda\rangle .
$$

The Wehrl entropy is obtained by replacing $\langle\lambda|\rho \ln \rho| \lambda\rangle$ with $\langle\lambda|\rho| \lambda\rangle \ln \langle\lambda|\rho| \lambda\rangle$. Introducing the Husimi distribution [16],

$$
H(q, p)=\langle\lambda|\rho| \lambda\rangle,
$$

one can write the Wehrl entropy as

$$
S_{W}=-\int \frac{d q d p}{2 \pi \hbar} H(q, p) \ln H(q, p) .
$$

Since the function $F(x)=-x \ln x$ is concave, it follows that

$$
S_{W}>S_{v N} \geq 0 .
$$

The equality $S_{W}=S_{v N}$ is impossible [32], and this means that $S_{W}$ is always nonzero even for a pure state.
The Husimi distribution $H(q, p)$ is the closest analog in quantum mechanics of the classical phase space distribution $f(q, p)$. One may be tempted to use instead the more well-known Wigner distribution [15],

$$
W(q, p)=\int_{-\infty}^{\infty} d y e^{-i p y / \hbar}\langle q+y / 2|\rho| q-y / 2\rangle,
$$

and define

$$
\tilde{S}_{W} \equiv-\int \frac{d q d p}{2 \pi \hbar} W(q, p) \ln W(q, p)
$$

However, $W(q, p)$ is not positive definite, and therefore its logarithm is not well defined everywhere. These two distributions are related by Gaussian smearing,

$H(q, p)=\frac{1}{\pi \hbar} \int d q^{\prime} d p^{\prime} e^{-\left(q-q^{\prime}\right)^{2} / \hbar-\left(p-p^{\prime}\right)^{2} / \hbar} W\left(q^{\prime}, p^{\prime}\right)$.

As is clear from this expression, the Husimi distribution smooths out localized fluctuations in a phase space volume $\Delta q \Delta p<\hbar / 2$. Such fluctuations are unphysical in that they do not bring about measurable consequences due to the uncertainty principle. Due in part to this loss of information, $S_{W}$ is always nonvanishing.

As an example, consider a one-dimensional harmonic oscillator with the Hamiltonian $H=\frac{p^{2}+q^{2}}{2}$. For the $n$th excited state, the Husimi distribution can be analytically computed as

$$
H(q, p)=\frac{1}{n !} e^{-H / \hbar}\left(\frac{H}{\hbar}\right)^{n} .
$$

Substituting this into (6), we find

$$
S_{W}=n+1+\ln n !-n \psi(n+1),
$$

where $\psi$ is the digamma function. Asymptotically, $S_{W} \approx \frac{1}{2} \ln n$. On the other hand, the Wigner distribution oscillates and becomes negative (except for the ground state $n=0$ ). Thus, the alternative definition (9) does not make sense. Note that the von Neumann entropy vanishes for all levels $n$ because they are pure states.

\section{WEHRL ENTROPY IN QCD}

Let us now consider the entropy of partons in $1+3$ dimensional QCD. Since quantum field theory is not commonly formulated in terms of state vectors $|\psi\rangle$ and a density matrix, it appears difficult to define and evaluate the von Neumann (entanglement) entropy $S_{v N}$ in a modelindependent way. (Such a construction is nevertheless possible within certain frameworks $[9,10,14]$, and we shall discuss one such model in a later section.) We thus turn to the Wehrl entropy. The QCD Wigner distribution 
$W\left(x, b_{\perp}, k_{\perp}\right)$ is the generalization of the collinear PDF to include dependences on transverse momentum $k_{\perp}$ and impact parameter $b_{\perp}$. They are defined by ( $\hbar=1$ in the following)

$$
x W_{q}^{ \pm}\left(x, b_{\perp}, k_{\perp}\right)=\int \frac{d z^{-} d^{2} z_{\perp}}{(2 \pi)^{3} 2 P^{+}} \int \frac{d^{2} \Delta_{\perp}}{(2 \pi)^{2}} e^{-i x P^{+} z^{-}-i k_{\perp} \cdot z_{\perp}}\left\langle P+\Delta_{\perp} / 2\left|\bar{q}\left(b_{\perp}+z / 2\right) U^{ \pm} q\left(b_{\perp}-z / 2\right)\right| P-\Delta_{\perp} / 2\right\rangle,
$$

for quarks and

$$
x W_{g}^{ \pm \pm}\left(x, b_{\perp}, k_{\perp}\right)=\int \frac{d z^{-} d^{2} z_{\perp}}{(2 \pi)^{3} P^{+}} \int \frac{d^{2} \Delta_{\perp}}{(2 \pi)^{2}} e^{-i x P^{+} z^{-}-i k_{\perp} \cdot z_{\perp}}\left\langle P+\Delta_{\perp} / 2\left|\operatorname{Tr}\left[F^{+\alpha}\left(b_{\perp}+z / 2\right) U^{ \pm} F_{\alpha}^{+}\left(b_{\perp}-z / 2\right) U^{ \pm}\right]\right| P-\Delta_{\perp} / 2\right\rangle
$$

for gluons. $|P\rangle$ is the single hadron state (usually the proton) with momentum $P^{\mu}$. $U^{ \pm}$is the staple-shaped fundamental Wilson line along the light-cone extending to $z^{-}= \pm \infty$. In the quark case, $W_{q}^{+}$and $W_{q}^{-}$are simply related by $P T$ transformation. In the gluon case, there are two distinct Wigner distributions, the Weiszäcker-Williams (WW) distribution $W_{W W}=W_{g}^{++}$and the dipole Wigner distribution $W_{\text {dip }}=W_{g}^{+-}$. The difference in the Wilson line configuration means that they contribute to different observables. For instance, $W_{\text {dip }}$ contributes to diffractive dijet production in $e p$ and $p A$ collisions [24,29].

The QCD Husimi distributions for quarks and gluons are defined by smearing the corresponding Wigner distributions in phase space $\left(b_{\perp}, k_{\perp}\right)$ [23]

$$
\begin{aligned}
& x H_{q}\left(x, b_{\perp}, k_{\perp}\right)=\frac{1}{\pi^{2}} \int d^{2} b_{\perp}^{\prime} d^{2} k_{\perp}^{\prime} e^{-\left(b_{\perp}-b_{\perp}^{\prime}\right)^{2} / \ell^{2}-\ell^{2}\left(k_{\perp}-k_{\perp}^{\prime}\right)^{2}} x W_{q}\left(x, b_{\perp}^{\prime}, k_{\perp}^{\prime}\right), \\
& x H_{W W / \text { dip }}\left(x, b_{\perp}, k_{\perp}\right)=\frac{1}{\pi^{2}} \int d^{2} b_{\perp}^{\prime} d^{2} k_{\perp}^{\prime} e^{-\left(b_{\perp}-b_{\perp}^{\prime}\right)^{2} / \ell^{2}-\ell^{2}\left(k_{\perp}-k_{\perp}^{\prime}\right)^{2}} x W_{W W / \text { dip }}\left(x, b_{\perp}^{\prime}, k_{\perp}^{\prime}\right),
\end{aligned}
$$

where $\ell$ is an arbitrary parameter with the dimension of length. Notice that the widths of the two Gaussians are inversely related such that the smearing is done in the minimum uncertainty region $\Delta b_{\perp} \Delta k_{\perp}=\frac{1}{2}$.

Unlike in quantum mechanics, a general proof of positivity of the QCD Husimi distributions $H_{q / W W / \text { dip }}$ is unfortunately not available. However, Refs. $[25,36]$ provided nontrivial examples in which the Husimi distribution is smooth and positive everywhere although the corresponding Wigner distribution oscillates between positive and negative values. (We shall see another example of this below.) We thus assume the positivity of the Husimi distribution as a working hypothesis and define the Wehrl entropy as a function of $x$,

$S_{W}(x) \equiv-\int d^{2} b_{\perp} d^{2} k_{\perp} x H\left(x, b_{\perp}, k_{\perp}\right) \ln x H\left(x, b_{\perp}, k_{\perp}\right)$,

where $H=H_{q / W W / \text { dip }}$. We opt to use $x H$ instead of $H$ since we are considering entropy per unit rapidity $Y=\ln 1 / x$. If the Wigner distribution turns out to be positive definite in some approximations or model calculations, we may as well define an entropy by

$\tilde{S}_{W}(x) \equiv-\int d^{2} b_{\perp} d^{2} k_{\perp} x W\left(x, b_{\perp}, k_{\perp}\right) \ln x W\left(x, b_{\perp}, k_{\perp}\right)$.
However, such a definition has limited applicability. Firstly, the Wigner distribution typically has a perturbative tail $W \sim 1 / k_{\perp}^{2}$ which makes the $k_{\perp}$ integral logarithmically divergent. While one may argue that this should be cut off by the resolution scale $Q^{2}$, a more serious problem is that most likely positivity is not preserved by the QCD evolution [22,23].

In the following, we arbitrarily neglect the overall prefactor of $x H$ and $x W$ inside the logarithm. This factor modifies the entropy only by an amount proportional to the collinear parton distribution function (PDF) $\int d^{2} b_{\perp} d^{2} k_{\perp} x H_{q, g}\left(x, b_{\perp}, k_{\perp}\right)=\int d^{2} b_{\perp} d^{2} k_{\perp} x W_{q, g}\left(x, b_{\perp}, k_{\perp}\right)=$ $x q(x), x g(x)$. It thus does not carry nontrivial information about the phase space structure of partons.

As a trivial example, consider a free electron or a quark moving in the positive $z$ direction. The Wigner and Husimi distributions are (setting $x=1$ )

$$
\begin{aligned}
W\left(b_{\perp}, k_{\perp}\right) & =\delta^{(2)}\left(b_{\perp}\right) \delta^{(2)}\left(k_{\perp}\right), \\
H\left(b_{\perp}, k_{\perp}\right) & =\frac{e^{-b_{\perp}^{2} / \ell^{2}-\ell^{2} k_{\perp}^{2}}}{\pi^{2}} .
\end{aligned}
$$

While the Wigner distribution is positive, its logarithm does not make sense. The Wehrl entropy can be evaluated from the Husimi distribution as 


$$
S_{W}=\frac{1}{\pi^{2}} \int d^{2} b_{\perp} d^{2} k_{\perp} e^{-b_{\perp}^{2} / \ell^{2}-\ell^{2} k_{\perp}^{2}}\left(\frac{b_{\perp}^{2}}{\ell^{2}}+\ell^{2} k_{\perp}^{2}\right)=2 .
$$

The nonvanishing value reflects our inability to precisely determine position and momentum simultaneously due to the uncertainty principle.

\section{WEHRL ENTROPY OF SMALL-x GLUONS}

In this section, we shall focus on the Wehrl entropy generated by gluons in the small- $x$ region or, equivalently, the large rapidity region $Y=\ln \frac{1}{x} \gg 1$. As already mentioned in the Introduction, the number of gluons grows rapidly as $x$ is decreased, and these gluons show collective behaviors which may be treated semiclassically. It is thus very interesting to consider the Wehrl entropy of such states.

As recently shown in [24], at small $x$ the dipole Wigner distribution takes the following simple form,

$$
\begin{aligned}
x W_{\mathrm{dip}}\left(x, b_{\perp}, k_{\perp}\right)= & \frac{2 N_{c}}{\alpha_{S}(2 \pi)^{2}} \int \frac{d^{2} r_{\perp}}{(2 \pi)^{2}} e^{i k_{\perp} \cdot r_{\perp}}\left(\frac{1}{4} \nabla_{b_{\perp}}^{2}+k_{\perp}^{2}\right) \\
& \times \mathcal{S}\left(x, b_{\perp}, r_{\perp}\right),
\end{aligned}
$$

where $\mathcal{S}$ is the forward $\mathrm{S}$ matrix of a dipole of size $r_{\perp}$ at impact parameter $b_{\perp}$ scattering off the hadron of interest. In general, $W_{\text {dip }}$ is not positive definite due to the $b_{\perp^{-}}$ derivative term. An equally simple, general expression of the WW Wigner distribution is not available, but in a quasiclassical approximation, one can deduce the following form [37,38],

$$
x W_{W W}\left(x, b_{\perp}, k_{\perp}\right)=\frac{N_{c}^{2}-1}{4 \pi^{4} \alpha_{S} N_{c}} \int d^{2} r_{\perp} e^{i k_{\perp} \cdot r_{\perp}} \frac{1}{r_{\perp}^{2}}(1-\tilde{\mathcal{S}}),
$$

where again $\tilde{\mathcal{S}}=\tilde{\mathcal{S}}\left(x, b_{\perp}, k_{\perp}\right)$ is the dipole $\mathrm{S}$ matrix in the adjoint representation. After integrating over $b_{\perp}$, one can reproduce the WW unintegrated gluon distribution [37,38].

Let us evaluate these expressions in a GBW-like model [39]

$$
\mathcal{S}=e^{-\frac{1}{4} r_{\perp}^{2} Q_{s}^{2}\left(x, b_{\perp}\right)}, \quad \tilde{\mathcal{S}}=e^{-\frac{1}{4} r_{\perp}^{2} Q_{s g}^{2}\left(x, b_{\perp}\right)},
$$

where $Q_{s(g)}$ is the quark (gluon) saturation momentum which we assume to be of the form $Q_{s(g)}^{2}\left(x, b_{\perp}\right)=$ $\Lambda^{2}\left(\frac{1}{x}\right)^{\alpha} h_{(g)}\left(b_{\perp}^{2} \Lambda^{2}\right)$. ( $\Lambda$ is the confinement scale.) Inserting (23) into (22), we find

$$
\begin{aligned}
x W_{W W}\left(x, b_{\perp}, k_{\perp}\right)= & \frac{N_{c}^{2}-1}{4 \pi^{4} \alpha_{s} N_{c}} \\
& \times \int \frac{d^{2} r_{\perp}}{r_{\perp}^{2}} e^{i k_{\perp} \cdot r_{\perp}}\left(1-e^{-\frac{1}{4} Q_{s g}^{2}\left(x, b_{\perp}\right) r_{\perp}^{2}}\right) \\
= & \frac{N_{c}^{2}-1}{4 \pi^{3} \alpha_{s} N_{c}} \Gamma\left[0, \frac{k_{\perp}^{2}}{Q_{s g}^{2}\left(x, b_{\perp}\right)}\right]
\end{aligned}
$$

where $\Gamma[0, z]$ is the incomplete gamma function. Note that (24) is positive definite. While this may seem natural in view of the fact that the WW unintegrated gluon distribution admits a probabilistic interpretation, we emphasize that the positivity of $W_{W W}$ is not guaranteed in general and likely to be violated by the quantum evolution. Anyway, since (24) is positive, we can adopt the simpler definition (18) and obtain

$$
\begin{aligned}
\tilde{S}_{W} \equiv & -\int d^{2} b_{\perp} d^{2} k_{\perp} x W\left(x, b_{\perp}, k_{\perp}\right) \ln x W\left(x, b_{\perp}, k_{\perp}\right) \\
\simeq & -\frac{N_{c}^{2}-1}{4 \pi^{3} \alpha_{s} N_{c}} \int d^{2} k_{\perp} d^{2} b_{\perp} \Gamma\left[0, \frac{k_{\perp}^{2}}{Q_{s g}^{2}\left(x, b_{\perp}\right)}\right] \\
& \times \ln \Gamma\left[0, \frac{k_{\perp}^{2}}{Q_{s g}^{2}\left(x, b_{\perp}\right)}\right] \\
= & 0.248 \frac{N_{c}^{2}-1}{4 \pi \alpha_{s} N_{c}} \int_{0}^{\infty} d b_{\perp}^{2} Q_{s g}^{2}\left(Y, b_{\perp}^{2}\right) .
\end{aligned}
$$

We see that the entropy grows exponentially in rapidity $\tilde{S}_{W} \sim$ $Q_{s}^{2}(Y) \sim e^{\alpha Y}$ in this model. This is essentially due to the transverse dynamics of QCD and is also a consequence of geometric scaling which holds perfectly for the model at hand [that is, $x W\left(x, q_{\perp}\right)$ depends only on the ratio $\left.q_{\perp}^{2} / Q_{s}^{2}(Y)\right]$. The parametric dependence $\tilde{S}_{W} \propto C_{F} Q_{s}^{2} / \alpha_{s}$ agrees with the previous results in $[6,8,9]$ using other definitions of entropy. For a large nucleus with atomic number $A, \tilde{S}_{W} \sim \int d^{2} b_{\perp} Q_{s}^{2} \propto A^{2 / 3} A^{1 / 3}=A$. This is because the number of gluons in a large nucleus is additive in the quasiclassical approximation [40] and is consistent with the fact that the entropy is an extensive variable.

Next, we turn to the dipole Wigner distribution. Using the same Gaussian ansatz (23), it is evaluated as

$$
x W_{\text {dip }}\left(x, b_{\perp}, k_{\perp}\right)=\frac{2 N_{c}}{\alpha_{s}(2 \pi)^{2}}\left(\frac{\partial}{\partial b_{\perp}^{2}} b_{\perp}^{2} \frac{\partial}{\partial b_{\perp}^{2}}+k_{\perp}^{2}\right) \frac{e^{-\frac{k_{\perp}^{2}}{Q_{s}^{2}}}}{\pi Q_{s}^{2}} .
$$

For realistic profile functions $Q_{s}^{2}\left(b_{\perp}\right)$, we find that (26) is not positive definite. This is related to the fact that the dipole distribution does not have a probabilistic interpretation due to the nontrivial gauge link dependence. We thus compute instead the Husimi distribution (16): 


$$
\begin{aligned}
x H\left(x, b_{\perp}, k_{\perp}\right)= & \frac{2 N_{c}}{\ell^{4} \alpha_{s} \pi^{2}(2 \pi)^{2}} \int d^{2} b_{\perp}^{\prime} e^{-\left(b_{\perp}-b_{\perp}^{\prime}\right)^{2} / \ell^{2}-\frac{\ell^{2}}{1+\ell^{2} Q_{s}^{2}} k_{\perp}^{2}} \\
& \times\left[\frac{\left(b_{\perp}-b_{\perp}^{\prime}\right)^{2}}{\ell^{2}}+\frac{\left(\ell^{2} Q_{s}^{2}\right)^{2}}{\left(1+\ell^{2} Q_{s}^{2}\right)^{2}} \ell^{2} k_{\perp}^{2}\right. \\
& \left.-\frac{1}{1+\ell^{2} Q_{s}^{2}}\right] \frac{\ell^{2}}{1+\ell^{2} Q_{s}^{2}} .
\end{aligned}
$$

For an arbitrary function $Q_{s}^{2}\left(b_{\perp}\right)$ which is monotonically decreasing with increasing $b_{\perp},(27)$ is positive definite. To see this, note that $\frac{1}{1+\ell^{2} Q_{0}^{2}} \leq \frac{1}{1+\ell^{2} Q_{s}^{2}}<1 \quad$ where $Q_{0}^{2} \equiv Q_{s}^{2}\left(b_{\perp}=0\right)$. We then find

$$
\begin{aligned}
x H\left(x, b_{\perp}, k_{\perp}\right)> & \frac{2 N_{c} e^{-\ell^{2} k_{\perp}^{2}}}{\ell^{2} \alpha_{s} \pi^{2}(2 \pi)^{2}\left(1+\ell^{2} Q_{0}^{2}\right)} \\
& \times \int d^{2} b_{\perp}^{\prime} e^{-\frac{\left(b_{\perp}-b_{\perp}^{\prime}\right)^{2}}{\ell^{2}}}\left[\frac{\left(b_{\perp}-b_{\perp}^{\prime}\right)^{2}}{\ell^{2}}-1\right]=0 .
\end{aligned}
$$

We can thus safely compute the Wehrl entropy (17). For large values of $Q_{s}^{2}, x H\left(x, b_{\perp}, k_{\perp}\right)$ depends on $k_{\perp}$ only through the ratio $k_{\perp}^{2} / Q_{s}^{2}$. It is then clear that the entropy behaves as $S_{W} \propto N_{c} Q_{s}^{2} / \alpha_{s} \sim e^{\alpha Y}$.

Before leaving this section, we note that one can also consider the entropy of small- $x$ quarks. The sea quark distribution has been computed in the small- $x$ formalism [41-43]. In a quasiclassical approximation, one can introduce the $b_{\perp}$ dependence in these results as

$$
\begin{aligned}
x W_{q}\left(x, b_{\perp}, k_{\perp}\right)= & \frac{N_{c}}{4 \pi^{4}} \int d^{2} k_{g \perp} F\left(x, k_{g \perp}, Q_{s}^{2}\left(b_{\perp}\right)\right) \\
& \times\left(1-\frac{k_{\perp} \cdot\left(k_{\perp}-k_{g \perp}\right)}{k_{\perp}^{2}-\left(k_{\perp}-k_{g \perp}\right)^{2}}\right. \\
& \left.\times \ln \frac{k_{\perp}^{2}}{\left(k_{\perp}-k_{g \perp}\right)^{2}}\right),
\end{aligned}
$$

where $F\left(k_{g \perp}\right)$ is the Fourier transform of $\mathcal{S}\left(r_{\perp}\right)$. As already mentioned, this has a perturbatve tail $W_{q} \sim 1 / k_{\perp}^{2}$. One can eliminate this tail by switching to the Husimi distribution and find $S_{W} \propto N_{c} Q_{s}^{2}$. Thus, the entropy of quarks is smaller than that of gluons by a factor of $\alpha_{s}$, as expected.

\section{TOWARDS THE SATURATION OF ENTROPY}

The results in the previous section suggest that entropy grows indefinitely as the rapidity $Y=\ln 1 / x$ is increased. However, we do not believe that this rapid growth continues forever. In this respect, it may be useful to draw an analogy to the classical entropy Eq. (1) of a time-dependent system. $S_{c l}(t)$ grows monotonically and eventually reaches a plateau as the system equilibrates. When this occurs, the collision term of the Boltzmann equation vanishes because the "gain" terms are exactly canceled by the "loss" terms. In QCD, the rapidity $Y=\ln 1 / x$ plays the role of time, and the rapid growth of entropy with $Y$ is essentially because one has included only the gain terms, namely, gluon splittings. By including the loss terms, or gluon recombinations, the number of gluons eventually saturates, and so does the entropy.

Unfortunately, a complete treatment of both the splitting and recombination effects, or the Pomeron loop effect, is an unsolved open question. Here we adopt a simple $1+0$ dimensional model which was originally introduced in the context of Mueller's dipole model [44]. Since there is no transverse phase space in $1+0$ dimensions, the Wehrl entropy cannot be defined. Still, in this model, one can naturally introduce the density matrix and calculate the von Neumann entropy as was done recently in [10]. We thus study the effect of Pomeron loops on the von Neumann entropy with the hope of gaining some insights into the fate of entropy in actual QCD.

Let $P_{n}(Y)$ be the probability to find $n$ dipoles (gluons) at time $Y$ starting from a single dipole at $Y=0 . P_{n}$ satisfies the equation

$$
\frac{d}{d Y} P_{n}=-\alpha n P_{n}+\alpha(n-1) P_{n-1} .
$$

This equation only describes gluon splittings, and $\alpha>0$ is the corresponding probability. Defining the generating function,

$$
Z(Y, u)=\sum_{n=1}^{\infty} P_{n}(Y) u^{n},
$$

one can show that (30) is equivalent to the following equation,

$$
\frac{d}{d Y} Z=\alpha\left(Z^{2}-Z\right), \quad Z(Y=0)=u
$$

which can be easily solved as

$$
Z=\frac{u}{u+(1-u) e^{\alpha Y}}
$$

It immediately follows that

$$
P_{n}=\left.\frac{1}{n !} \frac{d^{n} Z}{d u^{n}}\right|_{u=0}=e^{-\alpha Y}\left(1-e^{-\alpha Y}\right)^{n-1} .
$$

The von Neumann entropy for this system is defined [see (3)] and calculated as

$$
S_{v N}=-\sum_{n} P_{n} \ln P_{n} \approx \alpha Y=\ln \langle n\rangle,
$$

at large- $Y$. Thus, the entropy grows linearly with $Y$ or, equivalently, logarithmically with the average multiplicity in this model [10].

Generalization to $1+3$-dimensional QCD is significantly more complicated. The $n$-dipole probability $P_{n}$ 

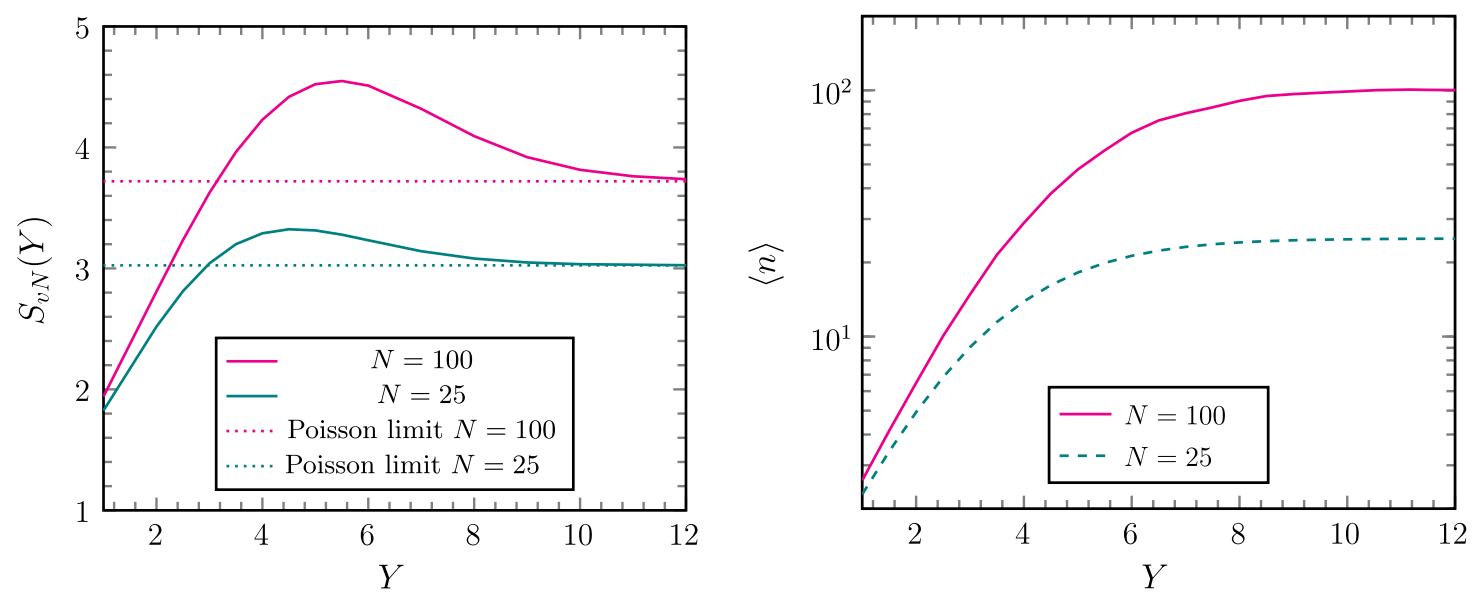

FIG. 1. von Neumann entropy $S_{v N}$ and $\langle n\rangle$ as a function of rapidity $Y$. We have set $\alpha=1$ and used (45) in (35). The region $\alpha Y \lesssim 1$ is excluded because our approximatios which led to (45) are not valid there.

now depends on $n$ two-dimensional vectors $\left\{z_{\perp}\right\}$ specifying the size and impact parameter of dipoles. One then defines

$S_{v N}=-\sum_{n}^{\infty} \int \prod_{i}^{n} d^{2} z_{\perp i} P_{n}\left(Y,\left\{z_{\perp}\right\}\right) \ln P_{n}\left(Y,\left\{z_{\perp}\right\}\right)$.

It seems very hard to evaluate this in full generality. In [10], the authors used several approximations and obtained $S_{v N} \sim\left(\ln Q_{s}^{2}(Y)\right) Y \propto Y^{2}$, which however does not agree with the behavior $S_{W} \sim Q_{s}^{2} \sim e^{Y}$ found in the previous section and also in $[6,8,9]$. This is simply due to different definitions of entropy. Nevertheless, it is interesting to point out a structural similarity between the two definitions: In the dipole approach, and in a frame in which the target dipole is slowly moving, the $\mathrm{S}$ matrix and $P_{n}$ are linearly related as (see, e.g., [45])

$\mathcal{S}\left(Y, b_{\perp}, r_{\perp}\right)=\sum_{n} \int \prod_{i}^{n} d^{2} z_{\perp i} P_{n}\left(Y,\left\{z_{\perp}\right\}\right) s_{0} s_{1} \ldots s_{n}$

where $s_{i}=s_{i}\left(z_{\perp i}, z_{\perp i+1}\right)$, with $z_{\perp 0}=b_{\perp}+\frac{r_{\perp}}{2}, z_{\perp n}=b_{\perp}-\frac{r_{\perp}}{2}$, is the $\mathrm{S}$ matrix of an elementary dipole off the target. Therefore, roughly we have $S_{W} \sim \mathcal{S} \ln \mathcal{S} \sim P_{n} \ln P_{n}$. The difference, then, appears to be attributed to an additional integration over the impact parameter $b_{\perp}$ in (17) which, by dimensional reasons, brings in a factor $Q_{s}^{2}{ }^{1}$

Returning to the $1+0$-dimensional problem, we now discuss the saturation of entropy by including the

\footnotetext{
${ }^{1}$ In Ref. [10], it was assumed that $P_{n}\left(\left\{z_{\perp}\right\}\right)$ was a function only of the absolute value of dipole sizes $\left|z_{\perp i}-z_{\perp i+1}\right|$ and not of their impact parameter.
}

recombination effect. ${ }^{2}$ Following [46,47], we generalize (30) as

$$
\begin{aligned}
\frac{d}{d Y} P_{n}= & -\alpha n P_{n}+\alpha(n-1) P_{n-1}+\beta n(n+1) P_{n+1} \\
& -\beta n(n-1) P_{n},
\end{aligned}
$$

with $\beta=\alpha_{s}^{2} \alpha>0$ and $\alpha_{s}^{2} \ll 1$. The last two terms represent the $2 \rightarrow 1$ recombination process with probability $\beta$. As shown in [47], Eq. (38) admits a stationary solution $\left(P_{n}\right.$ independent of $Y$ ) which is Poissonian,

$$
P_{n}=\frac{N^{n}}{n !} e^{-N}
$$

where $N \equiv 1 / \alpha_{s}^{2}=\langle n\rangle$. This already indicates that the entropy will saturate eventually. In order to study the preasymptotic behavior, it is convenient to consider the moments

$n^{(k)} \equiv \sum_{n} n(n-1) \cdots(n-k+1) P_{n}=\left.\frac{d^{k}}{d u^{k}} Z(u)\right|_{u=1}$,

where $Z$ is as defined in (31). The equation for $n^{(k)}$ reads

$$
\begin{aligned}
\frac{d}{d Y} n^{(k)}= & k \alpha n^{(k)}+k(k-1) \alpha n^{(k-1)} \\
& -k \beta n^{(k+1)}-\beta k(k-1) n^{(k)} .
\end{aligned}
$$

\footnotetext{
${ }^{2}$ To avoid confusion, we note that the saturation and unitarity of scattering amplitudes is achieved already in the model (30) (and its $1+3$-dimensional generalization) [44], as suggested by the nonlinear term in (32). This is a consequence of the "duality" of high energy evolution: a splitting in the projectile can be viewed as a recombination in the target. The genuine recombination effect in the projectile is missing.
} 
An approximate perturbative solution for $n^{(k=1)}$, neglecting terms of order $\mathcal{O}\left(\alpha_{s}^{2} \alpha Y\right)$ and $\mathcal{O}\left(e^{-\alpha Y}\right)$, has been obtained in [46] (see Eq. (13) there). It is straightforward to generalize this result to arbitrary $k$. We find

$$
n^{(k)} \approx \frac{e^{k \alpha Y}}{\Gamma(k)} \sum_{i=0}^{\infty}(-X)^{i} \frac{\Gamma(k+i+1) \Gamma(i+k)}{\Gamma(i+1)},
$$

where $X \equiv \alpha_{s}^{2} e^{\alpha Y}$. Physically, the index $i$ represents the number of Pomeron loop insertions. The series (42) has zero convergence radius, but is Borel summable. Using the identity $\Gamma(k)=\int_{0}^{\infty} d z z^{k-1} e^{-z}$, we can cast the above equation into

$$
n^{(k)} \approx e^{k \alpha Y} \int_{0}^{\infty} d z \frac{z^{k}}{(1+z X)^{k}} e^{-z}
$$

This allows us to reconstruct the generating function,

$$
Z(u)=\sum_{k=0}^{\infty} \frac{1}{k !} n^{(k)}(u-1)^{k}=\int_{0}^{\infty} d z e^{-z+\frac{N_{z} X}{1+z X}(u-1)},
$$

where $N \equiv \frac{1}{\alpha_{s}^{2}}$. We thus arrive at

$P_{n}=\left.\frac{1}{n !} \frac{d^{n} Z}{d u^{n}}\right|_{u=0}=\frac{N^{n}}{n !} \int_{0}^{\infty} d z e^{-z-\frac{N z X}{1+z X}}\left[\frac{z X}{1+z X}\right]^{n}$.

It is easy to check that $\sum_{n=0}^{\infty} P_{n}=1$. In Fig. 1 , we show the von Neumann entropy numerically computed from (45). Interestingly, the entropy is not monotonic although the average multiplicity $\langle n\rangle$ is. This indicates that $S_{v N}$ depends not only on $\langle n\rangle$ but also on higher moments. $S_{v N}(Y)$ takes a maximal value when $X=\alpha_{s}^{2} e^{\alpha Y} \sim 1$ and then starts to decrease and eventually saturates as $Y \rightarrow \infty$ to the known value of the Poisson distribution:

$$
S_{v N} \approx \frac{1}{2} \ln (2 \pi e N)-\frac{1}{12 N}+\mathcal{O}\left(1 / N^{2}\right) .
$$

Note that the coefficient in front of the logarithm $S_{v N} \sim$ $c \ln \langle n\rangle$ has changed from $c=1$ in (35) to $c=1 / 2$ [see also (12)], and this largely accounts for the decrease of entropy at $X>1$. $^{3}$

This nonmonotonic behavior of entropy seems counterintuitive at first sight, but after all there is no "H-theorem" for this quantity. ${ }^{4}$ Given that the von Neumann entropy measures the deviation from a pure state, we may say that the saturated gluon states probed at asymptotic energy are more ordered, in analogy to the terminology commonly used in condensed matter physics. In other words, the transition to the saturation resembles a phase transition,

\footnotetext{
${ }^{3}$ Incidentally, the aforementioned peak can disappear when $N<2 \pi e$, since $\frac{1}{2} \ln (2 \pi e N)$ becomes larger than $\ln N$. We only focus on the case where $N$ is sufficiently large.

${ }^{4}$ A similar nonmonotonic behavior of the Wehrl entropy has been observed in a different context $[34,35]$.
}

although it may not be a genuine phase transition, since we are considering the quantum fluctuations of partons inside the wave function of a confined hadron. It is also interesting to note that the Poisson distribution is known as the maximum entropy distribution among the $\infty$-generalized binomial distributions with fixed mean $\langle n\rangle$.

At last, we would like to comment on some observation related to the above probability distribution in Eq. (45). When $X \rightarrow \infty, P_{n}$ reduces to the Poisson distribution (39), as expected, since it is the stationary fixed point of Eq. (38). As long as $N X=e^{\alpha Y}$ is sufficiently large, we can also take the limit $X \ll 1$ and see that Eq. (45) then reduces to

$$
P_{n}=\left(\frac{N X}{1+N X}\right)^{n} \frac{1}{1+N X}
$$

which is known as the geometric distribution with $\langle n\rangle=N X=e^{\alpha Y}$. In phenomenology, the so-called negative binomial distribution (NBD), which is defined with two parameters $\langle n\rangle$ and $k$ as

$$
P_{n}^{N B}=\frac{\Gamma[n+k]}{\Gamma[n+1] \Gamma[k]}\left(\frac{\langle n\rangle}{k+\langle n\rangle}\right)^{n}\left(\frac{k}{k+\langle n\rangle}\right)^{k},
$$

is often used to describe the multiplicity distribution in high energy collisions $[48,49]$, and it can be derived from the small- $x$ framework [50,51]. The geometric distribution is simply the special case of the NBD with $k=1$. It is also interesting to notice that NBD with arbitrary $k$ always has larger value of entropy as compared to the Poisson distribution with the same fixed value of $\langle n\rangle$. Their entropy becomes the same when $k \rightarrow \infty$, since NBD reduces to the Poisson distribution in that limit.

\section{CONCLUSIONS}

In this paper, we have introduced and studied the Wehrl entropy of a hadron/nucleus defined through the QCD Husimi distribution. It quantifies the complexity of the multiparton distribution in phase space $\left(b_{\perp}, k_{\perp}\right)$, and therefore it is a very interesting notion in the tomographic study of the nucleon. At small $x$, our result parametrically agrees with the different definitions of (entanglement) entropy discussed in $[6,8,9]$. Unlike in these previous works, however, the Wehrl entropy is given in terms of the gauge invariant matrix element of the quark and gluon field operators, and as such, it is not restricted to small- $x$ gluons.

The phenomenological implications of our result remain to be explored. It is often argued that the entropy is proportional to the final state multiplicity $d n / d Y$, and the result $S_{W} \propto Q_{s}^{2}(Y) \sim e^{\alpha Y}$ appears to be consistent with the exponential growth of multiplicity with $Y \sim \ln s$. Now that we have a model-independent definition of entropy, such a correspondence can be pursued also at low-energy (large- $x$ ) and/or in quark-dominated processes. Concerning the high-energy limit, our result in Sec. V suggests that the 
exponential growth will be tamed by the Pomeron loop effect, possibly leading to a nearly constant (in $Y$ ) multiplicity. But presumably this occurs at very high energy which has not been reached yet in modern accelerators.

\section{ACKNOWLEDGMENTS}

Y. Hatta thanks Dmitri Kharzeev, Cedric Lorce and Hidekazu Tsukiji for helpful conversations. B.X. and F. Y. acknowledge interesting discussions with
Volker Koch. Y. Hatta and F. Y. thank the Central China Normal University, where this work was completed, for hospitality. This material is based upon work supported by the U.S. Department of Energy, Office of Science, Office of Nuclear Physics, under Contract No. DE-AC02-05CH11231 and by the Natural Science Foundation of China (NSFC) under Grant No. 11575070. Y. Hagiwara is supported by the JSPS KAKENHI Grant No. 17J08072.
[1] E. Mac and E. Ugaz, Z. Phys. C 43, 655 (1989).

[2] R. S. Bhalerao, Phys. Lett. B 380, 1 (1996); 387, 881(E) (1996).

[3] C. Bourrely, J. Soffer, and F. Buccella, Eur. Phys. J. C 23, 487 (2002).

[4] S. Munier and R. B. Peschanski, Phys. Rev. Lett. 91, 232001 (2003).

[5] E. Iancu, A. H. Mueller, and S. Munier, Phys. Lett. B 606, 342 (2005).

[6] K. Kutak, Phys. Lett. B 705, 217 (2011).

[7] A. Stoffers and I. Zahed, Phys. Rev. D 88, 025038 (2013).

[8] R. Peschanski, Phys. Rev. D 87, 034042 (2013).

[9] A. Kovner and M. Lublinsky, Phys. Rev. D 92, 034016 (2015).

[10] D. E. Kharzeev and E. M. Levin, Phys. Rev. D 95, 114008 (2017).

[11] E. Shuryak and I. Zahed, arXiv:1707.01885.

[12] H. T. Elze, Nucl. Phys. B436, 213 (1995).

[13] H. T. Elze, Phys. Lett. B 369, 295 (1996).

[14] D. E. Miller, Eur. Phys. J. C 34, 435 (2004).

[15] E. Wigner, Phys. Rev. 40, 749 (1932).

[16] K. Husimi, Proc. Phys. Math. Soc. Jpn. 22, 264 (1940).

[17] A. V. Belitsky, X.d. Ji, and F. Yuan, Phys. Rev. D 69, 074014 (2004).

[18] S. Meissner, A. Metz, and M. Schlegel, J. High Energy Phys. 08 (2009) 056.

[19] C. Lorce and B. Pasquini, Phys. Rev. D 84, 014015 (2011).

[20] Y. Hatta, Phys. Lett. B 708, 186 (2012).

[21] C. Lorce, B. Pasquini, X. Xiong, and F. Yuan, Phys. Rev. D 85, 114006 (2012).

[22] A. Mukherjee, S. Nair, and V. K. Ojha, Phys. Rev. D 90, 014024 (2014).

[23] Y. Hagiwara and Y. Hatta, Nucl. Phys. A940, 158 (2015).

[24] Y. Hatta, B. W. Xiao, and F. Yuan, Phys. Rev. Lett. 116, 202301 (2016).

[25] Y. Hagiwara, Y. Hatta, and T. Ueda, Phys. Rev. D 94, 094036 (2016).

[26] T. Gutsche, V. E. Lyubovitskij, and I. Schmidt, Eur. Phys. J. C 77, 86 (2017).

[27] J. Zhou, Phys. Rev. D 94, 114017 (2016).
[28] S. Bhattacharya, A. Metz, and J. Zhou, Phys. Lett. B 771, 396 (2017).

[29] Y. Hagiwara, Y. Hatta, R. Pasechnik, M. Tasevsky, and O. Teryaev, Phys. Rev. D 96, 034009 (2017).

[30] J. More, A. Mukherjee, and S. Nair, arXiv:1709.00943.

[31] A. Rajan, M. Engelhardt, and S. Liuti, arXiv:1709.05770.

[32] A. Wehrl, Rep. Math. Phys. 16, 353 (1979).

[33] T. Kunihiro, B. Muller, A. Ohnishi, and A. Schafer, Prog. Theor. Phys. 121, 555 (2009).

[34] H. Tsukiji, H. Iida, T. Kunihiro, A. Ohnishi, and T. T. Takahashi, Phys. Rev. D 94, 091502 (2016).

[35] H. Tsukiji, T. Kunihiro, A. Ohnishi, and T. T. Takahashi, Prpg. Theor. Exp. Phys. 2018, 013D02 (2018).

[36] Y. Hatta and Y. Hagiwara, EPJ Web Conf. 112, 01010 (2016).

[37] Y. V. Kovchegov and A. H. Mueller, Nucl. Phys. B529, 451 (1998).

[38] F. Dominguez, C. Marquet, B. W. Xiao, and F. Yuan, Phys. Rev. D 83, 105005 (2011).

[39] K. J. Golec-Biernat and M. Wusthoff, Phys. Rev. D 59, 014017 (1998).

[40] A. H. Mueller, arXiv:hep-ph/9911289.

[41] L. D. McLerran and R. Venugopalan, Phys. Rev. D 59, 094002 (1999).

[42] C. Marquet, B. W. Xiao, and F. Yuan, Phys. Lett. B 682, 207 (2009).

[43] A. H. Mueller, Nucl. Phys. B558, 285 (1999).

[44] A. H. Mueller, Nucl. Phys. B437, 107 (1995).

[45] Y. Hatta, E. Iancu, C. Marquet, G. Soyez, and D. N. Triantafyllopoulos, Nucl. Phys. A773, 95 (2006).

[46] A. I. Shoshi and B. W. Xiao, Phys. Rev. D 73, 094014 (2006).

[47] S. Bondarenko, L. Motyka, A. H. Mueller, A. I. Shoshi, and B.-W. Xiao, Eur. Phys. J. C 50, 593 (2007).

[48] G. J. Alner et al. (UA5 Collaboration), Phys. Lett. 160B, 193 (1985).

[49] J. F. Grosse-Oetringhaus and K. Reygers, J. Phys. G 37, 083001 (2010).

[50] F. Gelis, T. Lappi, and L. McLerran, Nucl. Phys. A828, 149 (2009).

[51] A. Dumitru and E. Petreska, arXiv:1209.4105. 